ISSN 0103-5150

Fisioter. Mov., Curitiba, v. 24, n. 2, p. 239-246, abr./jun. 2011 Licenciado sob uma Licença Creative Commons

\title{
Exercício aeróbico durante a hemodiálise: relato de cinco anos de experiência
}

\author{
Aerobic exercise during hemodialysis: report of \\ five years experience
}

\section{Maycon de Moura Reboredo ${ }^{[a]}$, Ruiter de Souza Faria ${ }^{[b]}$, Leonardo Henriques Portes ${ }^{[c]}$, Caroline Gomes Mol ${ }^{[\mathrm{d}]}$, Bruno do Valle Pinheiro ${ }^{[\mathrm{e}]}$, Rogério Baumgratz de Paula ${ }^{[\mathrm{f}]}$}

[a] Fisioterapeuta, Doutor em Saúde pela Universidade Federal de Juiz de Fora (UFJF), professor do Instituto Federal de Educação, Ciência e Tecnologia do Sudeste de Minas Gerais, membro do Núcleo Interdisciplinar de Estudos e Pesquisas em Nefrologia (NIEPEN) da Universidade Federal de Juiz de Fora (UFJF), Juiz de Fora, MG - Brasil, e-mail: mayconreboredo@yahoo.com.br

[b] Fisioterapeuta, Mestrando em Saúde pela UFJF, membro do NIEPEN da Universidade Federal de Juiz de Fora (UFJF), Juiz de Fora, MG - Brasil, e-mail: ruiterfaria@gmail.com

[c] Fisioterapeuta, Mestrando em Saúde Coletiva pela Universidade Federal de Juiz de Fora (UFJF), Juiz de Fora, MG - Brasil, e-mail: leo.portes@yahoo.com.br

[d] Fisioterapeuta, membro do NIEPEN da Universidade Federal de Juiz de Fora (UFJF), Juiz de Fora, MG - Brasil, e-mail: carol_gmol@hotmail.com

[e] Pneumologista, Doutor em Pneumologia pela Universidade Federal de São Paulo, professor de Pneumologia e Semiologia da Faculdade de Medicina da Universidade Federal de Juiz de Fora (UFJF), Juiz de Fora, MG - Brasil, e-mail: bvalle@terra.com.br

[f] Nefrologista, Doutor em Nefrologia pela Universidade Federal de São Paulo, professor de Nefrologia da Faculdade de Medicina da UFJF, membro do NIEPEN da Universidade Federal de Juiz de Fora (UFJF), Juiz de Fora, MG - Brasil, e-mail: rbaumgratz@msn.com

\section{Resumo}

Introdução: Nos pacientes portadores de doença renal crônica (DRC) submetidos à hemodiálise (HD), o sedentarismo compromete a capacidade funcional e se associa a aumento da mortalidade por doenças cardiovasculares. Apesar da demonstração dos benefícios da prática de exercícios físicos para estes pacientes, poucos centros de diálise desenvolvem esta atividade. 0 objetivo deste estudo foi descrever a experiência de cinco anos de um programa de exercício aeróbico intradialítico, com ênfase na adesão e na segurança do 
procedimento. Métodos: Foram incluídos pacientes adultos, de ambos os sexos, que participaram de um programa de exercícios realizado no período de agosto de 2004 a agosto de 2009.0 exercício foi realizado nas duas horas iniciais da HD, empregando-se um cicloergômetro horizontal, sendo constituído de aquecimento, condicionamento e resfriamento. Foram realizadas análises descritivas dos dados coletados ao longo dos cinco anos do programa. Resultados: No período de cinco anos, 34 pacientes (48,26 \pm 11,44 anos; 20 mulheres) participaram do programa de exercício. Foram realizadas 3.077 sessões individuais de exercício aeróbico durante as sessões de HD. 0 tempo médio de participação no programa foi de 15,65 \pm 13,86 meses e a aderência às sessões de exercício foi de $64,87 \pm 19,47 \%$. Apesar do registro de alguns episódios de hipotensão, sem repercussões hemodinâmicas significativas, não foram observadas complicações clínicas importantes. Conclusão: A realização de exercício aeróbico durante a sessão de HD é uma prática bem aceita pelos pacientes, segura e não acompanhada de complicações graves.

Palavras-chave: Exercício. Falência renal crônica. Diálise renal.

\section{Abstract}

Introduction: A sedentary lifestyle has a negative impact on functional capacity being closely linked to cardiovascular mortality in patients with end-stage renal disease (ESRD) on hemodialysis (HD). In this population, an exercise training program has been associated several benefits. However, only a few dialysis units have adopted this program. The aim of this study was to describe a five year's experience of intradialytic exercise training, focused on adherence and safety of the procedure. Methods: The sample included adult patients of both genders who joined an exercise training program during HD sessions between August 2004 and August 2009. The training was done during the first two hours of HD sessions by using a stationary bicycle and consisted of warm-up, aerobic exercise and cool-down. Descriptive analyses of data collected throughout the five years of the program were done. Results: In five years, 34 patients ( $48.26 \pm 11.44$ years, 20 women) participated in the exercise training program completing 3077 individual sessions of exercise. The mean time of training was $15.65 \pm 13.86$ months and the adherence was of $64.87 \pm 19.47 \%$. Despite a few episodes of hypotension without significant hemodynamic repercussion we did not observe any major complications during the study. Conclusion: A program of exercise training during HD sessions is well accepted by the patients, is safe and not associated with serious complications.

Keywords: Exercise. Kidney failure chronic. Renal dialysis.

\section{Introdução}

Nos pacientes portadores de doença renal crônica (DRC), submetidos à hemodiálise (HD), as complicações cardiovasculares contribuem sobremaneira para a diminuição da capacidade funcional, para a baixa tolerância ao exercício e, consequentemente, para a dificuldade de realização das atividades de vida diária. Além disso, a redução da capacidade funcional nestes pacientes pode ser atribuída à uremia, à anemia, à atrofia e à fraqueza muscular, ao sedentarismo, à desnutrição, à alteração na regulação do potássio, entre outros (1-6).

Apesar da melhoria da qualidade de diálise, do melhor controle da anemia e de outras complicações inerentes à DRC, a capacidade funcional ainda permanece diminuída nos pacientes renais crônicos em HD (7).
Com isso, novas estratégias têm sido utilizadas para melhorar a capacidade funcional destes pacientes, como, por exemplo, o combate ao sedentarismo.

As alterações físicas secundárias à uremia, bem como o próprio processo de HD, são condições que favorecem o sedentarismo nos pacientes renais crônicos. Além de influenciar negativamente o sistema cardiovascular e a capacidade funcional, o sedentarismo está intimamente ligado com a mortalidade na DRC $(8,9)$. Neste sentido, O'Hare et al. (8) avaliaram dois grupos de pacientes renais crônicos em tratamento dialítico no intervalo de um ano e concluíram que pacientes sedentários apresentavam risco de morte $62 \%$ maior, quando comparados aos não sedentários. Sendo assim, vários estudos têm sido conduzidos aplicando um protocolo de exercício físico a estes pacientes. 
0 primeiro estudo que relata a realização de exercícios físicos em pacientes com DRC submetidos à HD foi conduzido em 1979 por Goldberg et al. (10). Neste estudo, os autores avaliaram o efeito de um programa de treinamento aeróbico, que não foi realizado durante as sessões de HD, nos fatores de risco para a doença arterial coronariana. Em 1986, Painter et al. (11) aplicaram o primeiro protocolo de exercício aeróbico durante as sessões de HD e observaram que seis meses de treinamento foi eficaz para aumentar o pico de consumo de oxigênio $\left(\mathrm{VO}_{2}\right.$ pico) e reduzir o uso de medicação anti-hipertensiva em alguns pacientes. A partir deste primeiro estudo, o interesse por esta área vem crescendo, principalmente nos últimos dez anos.

Alguns autores têm demonstrado que um programa de exercício aeróbico intradialítico proporciona vários benefícios. Na Tabela 1 estão demonstrados alguns dos efeitos do exercício aeróbico durante as sessões de HD nos impactos negativos da DRC.

Apesar dos benefícios do exercício físicos nos pacientes em HD já serem bastante difundidos, e de vários centros nos Estados Unidos e na Europa adotarem esta prática na rotina da HD, poucos centros no Brasil desenvolvem esta atividade.

0 objetivo do presente estudo foi descrever a experiência de cinco anos de um programa de exercício aeróbico intradialítico no setor de HD do Hospital Universitário da Universidade Federal de Juiz de Fora, MG, com ênfase na adesão e na segurança do procedimento.

\section{Materiais e métodos}

\section{Amostra}

A amostra foi constituída de pacientes que participaram do programa de exercício aeróbico realizado durante as sessões de HD no período de agosto de 2004 a agosto de 2009. Neste programa foram incluídos pacientes adultos, de ambos os sexos e que estavam em tratamento hemodialítico por um período mínimo de três meses.

Os critérios de exclusão foram pacientes com angina instável, arritmia não controlada, insuficiência cardíaca descompensada, hipertensão arterial descontrolada com pressão arterial sistólica (PAS) $\geq 200$ mmHg e/ou pressão arterial diastólica (PAD) $\geq 120$ $\mathrm{mmHg}$, pericardite ou miocardite aguda, diabetes mellitus descontrolada (glicemia de jejum $>300 \mathrm{mg}$ / dl), insuficiência ou estenose mitral ou aórtica graves sem tratamento adequado, pneumopatias graves, infecção sistêmica aguda, osteodistrofia renal grave,

Tabela 1 - Efeitos do exercício realizado durante as sessões de hemodiálise nos impactos negativos da doença renal crônica

\begin{tabular}{ll}
\hline Impactos da doença renal crônica & Efeito do exercício durante a hemodiálise \\
\hline Redução do $\mathrm{VO}_{2}$ pico & Aumento do $\mathrm{VO}_{2}$ pico $(4,11,12)$ \\
Redução do $\mathrm{VO}_{2}$ pico no limiar anaeróbico & Aumento do $\mathrm{VO}_{2}$ pico no limiar anaeróbico (12) \\
Elevação da pressão arterial & Redução da pressão arterial (11-14) \\
Utilização de anti-hipertensivos & Redução da utilização de anti-hipertensivos (14) \\
Concentração elevada de ureia e fosfato & Aumento da remoção de ureia e fosfato durante a hemodiálise (15) \\
Redução da hemoglobina & Aumento da hemoglobina $(10,13)$ \\
Redução da força e resistência muscular & Aumento da força e resistência muscular $(16,17)$ \\
Redução da capacidade funcional & Aumento da capacidade funcional $(4,13,17,18)$ \\
Aumento da sensação de fadiga & Redução da sensação de fadiga $(19)$ \\
Baixa qualidade de vida & Aumento da qualidade de vida $(4,13,17,18)$ \\
Aumento da ansiedade & Redução da ansiedade $(20)$ \\
Aumento do relato de dor & Redução das queixas álgicas $(18)$ \\
Redução da vitalidade & Aumento da vitalidade (18) \\
\hline
\end{tabular}

Nota: $\mathrm{VO}_{2}$ pico = pico de consumo de oxigênio.

Fonte: Dados da pesquisa. 
além de distúrbios neurológicos, musculoesqueléticos e osteoarticulares incapacitantes.

\section{Avaliações iniciais}

Todos os pacientes foram submetidos à avaliação cardiológica e fisioterapêutica, antes da inclusão no programa de exercício. A avaliação cardiológica foi composta de anamnese, exame físico, exames de eletrocardiograma e de ecocardiograma, além de teste cardiopulmonar. Esta avaliação teve como objetivo verificar a presença de alterações cardiovasculares que contraindicassem a execução dos exercícios. Na avaliação fisioterapêutica foram efetuados anamnese e exame físico, com o objetivo de verificar a existência de limitações osteomioarticulares que impedissem a realização dos exercícios.

\section{Treinamento aeróbico}

0 treinamento aeróbico foi supervisionado, realizado nas duas horas iniciais da HD, com duração média de uma hora. 0 treinamento foi composto de três etapas: aquecimento, condicionamento e resfriamento. Foi utilizado um cicloergômetro eletromagnético horizontal (Movement, BM 4000, Brudden Equipamentos Ltda, Pompeia, SP, Brasil) para a realização do exercício aeróbico.

No aquecimento foram realizados exercícios de alongamentos de membros inferiores por aproximadamente dez minutos, além de atividade aeróbica com a menor carga $(4,9 \mathrm{~N} \cdot \mathrm{m})$ e com baixa rotação (até 35 RPM), com duração de cinco minutos.

$\mathrm{Na}$ etapa de condicionamento foi realizado o exercício aeróbico por até 35 minutos. 0 tempo de exercício foi individual, de acordo com a resposta de cada paciente, que iniciaram com o tempo tolerado e foram estimulados a aumentar até completarem os 35 minutos. Os pacientes foram orientados a permanecerem com uma rotação constante durante todo o exercício aeróbico para que sua intensidade se mantivesse constante. A intensidade do treinamento foi determinada pela Escala de Percepção Subjetiva do Esforço de Borg, na qual os pacientes teriam que permanecer entre 11 (leve) a 13 (um pouco intenso) (21). A intensidade foi monitorada a cada cinco minutos de treinamento, e se o paciente indicasse um valor menor do que 11 na escala, ele era estimulado a aumentar a rotação, e se o valor na escala fosse maior do que 13, a diminuí-la. A carga foi prescrita de acordo com a tolerância de cada paciente para a manutenção da intensidade entre 11 e 13 na escala de Borg durante todo o período de exercício, e foi aumentada quando o paciente atingiu rotação maior do que 60 RPM.

No resfriamento foi realizado de um a três minutos de exercício aeróbico com a carga de 4,9 N.m e com rotação baixa.

A pressão arterial foi monitorada no repouso, a cada cinco minutos do condicionamento e após o resfriamento. A frequência cardíaca foi monitorada continuamente por meio de um cardiofrequencímetro (Polar F1, Polar Electro Oy, Kempele, Finland).

Os critérios para interrupção do exercício aeróbico incluíam cansaço físico intenso (escala de Borg $\geq 15$ ), dor torácica, hipoglicemia, vertigem, palidez, lipotímia, pré-síncope, dispneia desproporcional à intensidade do esforço, alterações significativas da frequência cardíaca, da PAS e/ou PAD (baseado nos níveis iniciais e no resultado do teste cardiopulmonar) e fadiga de membros inferiores.

Quando os pacientes apresentaram alteração de pressão arterial (PAS > $180 \mathrm{mmHg}$ e/ou PAD >110 $\mathrm{mmHg}$ ), glicemia pós-prandial $<90 \mathrm{mg} / \mathrm{dl}$, ganho de peso interdialítico maior do que $5 \mathrm{~kg}$, dificuldade no acesso vascular e alguma queixa significativa (dor, dispneia, etc.) antes do treinamento, eles foram impedidos de realizar o exercício nesse dia ou enquanto persistiram tais alterações.

\section{Variáveis estudadas e análise estatística}

Procedeu-se à análise dos prontuários dos pacientes no período de agosto de 2004 a agosto de 2009. Obtiveram-se dos prontuários as seguintes variáveis: características demográficas e clínicas dos pacientes, número de sessões, motivos de não realização, complicações e causas de interrupção do exercício aeróbico e causas de abandono do programa. Foram realizadas análises descritivas dos dados por meio do programa SPSS ${ }^{\circledR} 11.0$.

\section{Aspectos éticos}

Durante os cinco anos do protocolo, foram realizados três projetos de pesquisa. Os projetos foram apro- 
vados pelo Comitê de Ética em Pesquisa da Universidade Federal de Juiz de Fora e todos os pacientes que concordaram em participar dos estudos assinaram o Termo de Consentimento Livre e Esclarecido.

\section{Resultados}

\section{Pacientes}

Neste período de cinco anos, 34 pacientes participaram do programa de exercício aeróbico realizado durante as sessões de HD. Na Tabela 2 estão descritas as características demográficas e clínicas dos pacientes.

\section{Treinamento aeróbico}

Foram realizadas 3.077 sessões individuais de exercício aeróbico durante as sessões de HD. Cada paciente realizou em média $90,5 \pm 73,22$ sessões de exercício. Considerando todos os pacientes, a aderência ao exercício foi de $64,87 \pm 19,47 \%$. As causas da não realização do exercício estão descritas na Tabela 3.

Não foram observadas complicações importantes com a realização do exercício. Apenas um paciente apresentou dois episódios de hipotensão severa durante a atividade. As causas de interrupção do exercício podem ser vistas na Tabela 4 .

Os pacientes realizaram em média 15,65 $\pm 13,86$ meses de treinamento aeróbico. A principal causa de abandono do programa de exercício foi a falta de motivação, perfazendo um total de $40 \%$ dos pacientes. As outras causas mais prevalentes foram complicações cardiovasculares (20\%), limitações por outras patologias, como osteoartrose (15\%), e realização de transplante renal (10\%).

\section{Discussão}

Neste estudo, observamos que o exercício aeróbico realizado durante as sessões de HD mostrouse uma prática bem aceita pelos pacientes, segura e não associada à intercorrências clínicas relevantes.

A aderência média ao exercício realizado durante as sessões de HD no presente estudo foi de $65 \%$. Considerando que o valor médio de treinamento foi de 15 meses, acreditamos que os pacientes apresentaram boa aderência ao exercício. Resultados semelhantes foram demonstrados por Miller et al. (14), que aplicaram seis meses de exercício aeróbico durante as sessões de HD e observaram aderência de $56 \%$. Contrariamente, Moug et al. (20) aplicaram apenas 12 sessões de exercício durante a HD em seis semanas e observaram aderência de 99\%. Portanto,

Tabela 2 - Características demográficas e clínicas dos 34 pacientes que participaram do treinamento aeróbico

\begin{tabular}{lc}
\hline Características & Pacientes $(\mathbf{n}=\mathbf{3 4})$ \\
\hline Idade (anos) & $48,26 \pm 11,44$ \\
Sexo (masculino/feminino) & $14 / 20$ \\
Etnia (brancos/negros) & $16 / 18$ \\
Tempo de HD (meses) & $59,85 \pm 46,19$ \\
\hline Etiologia da DRC, pacientes (\%) & \\
\hline Glomerulonefrite crônica & $11(32,34 \%)$ \\
HA & $9(26,46 \%)$ \\
DM & $1(2,94 \%)$ \\
Uropatia obstrutiva & $3(8,82 \%)$ \\
Amiloidose renal & $2(5,88 \%)$ \\
Outras & $8(23,52 \%)$ \\
\hline
\end{tabular}

Nota: $\mathrm{HD}=$ hemodiálise, $\mathrm{DRC}=$ doença renal crônica, $\mathrm{HA}=$ hipertensão arterial, $\mathrm{DM}=$ diabetes mellitus. Fonte: Dados da pesquisa. 
Tabela 3 - Causas de não realização do exercício aeróbico

\begin{tabular}{lc}
\hline Causas de não realização: & Casos (\%) \\
\hline Quadro álgico & $281(18,78 \%)$ \\
Indisposição ou falta de motivação & $266(17,78 \%)$ \\
Alteração da pressão arterial & $214(14,3 \%)$ \\
Ganho excessivo de peso no período interdialítico & $153(10,23 \%)$ \\
Relato de cansaço físico & $133(8,89 \%)$ \\
Menstruação & $93(6,22 \%)$ \\
Dificuldade na punção & $38(2,54 \%)$ \\
Outros & $318(21,26 \%)$ \\
\hline
\end{tabular}

Fonte: Dados da pesquisa.

Tabela 4 - Causas de interrupção do exercício aeróbico

\begin{tabular}{ll}
\hline \multicolumn{1}{c}{ Causas de interrupção } & Casos (\%) \\
\hline Elevação excessiva da pressão arterial & $32(19,87 \%)$ \\
Relato de cansaço intenso & $26(16,15 \%)$ \\
Queda sustentada da pressão arterial & $18(11,18 \%)$ \\
Dor em membros inferiores & $15(9,32 \%)$ \\
Dispneia desproporcional à intensidade do esforço & $14(8,69 \%)$ \\
Outros & $56(34,78 \%)$ \\
\hline
\end{tabular}

Fonte: Dados da pesquisa.

pacientes renais crônicos tendem a aderir melhor aos protocolos de menor duração. Além disso, outro fator associado à aderência é a idade dos pacientes.

Dados da Sociedade Brasileira de Nefrologia mostram que no Brasil, a faixa etária da maioria dos pacientes com DRC em diálise é de 40 a 59 anos, o que está de acordo com a média de idade encontrada em nosso estudo que foi de 48 anos (22). A relação entre a idade dos pacientes em HD e a aderência a programas de exercício foi descrita por Daul et al. (23). Estes autores observaram que os pacientes com idade mais avançada foram mais aderentes às sessões de exercício em relação aos mais jovens. Graças à debilidade física e funcional dos pacientes mais idosos, a aderência ao exercício tende a ser maior, na tentativa de melhorar seu estado geral de saúde, o que não se observa nos pacientes hemodialisados mais jovens (23).

Um fator digno de nota é o estado de saúde dos pacientes com DRC em HD que também contribuiu para a aderência encontrada no nosso estudo. Em algumas sessões, os pacientes apresentaram contraindicação para realização do exercício durante a HD. Dentre as causas da não realização de exercícios citam-se a presença de queixas álgicas e de dispneia, níveis pressóricos elevados, hipotensão intradialítica, ganho excessivo de peso no período interdialítico e dificuldade no acesso vascular. Além desses, a falta de motivação contribuiu de modo significativo para a redução da aderência ao exercício.

No presente estudo, a falta de motivação também representou a principal causa de abandono do programa de exercício. Da mesma forma, Kouidi et al. (12) descreveram que a falta de motivação contribui sobremaneira para o abandono dos programas de exercícios nos pacientes portadores de DRC em diálise. Embora a falta de motivação tenha sido a principal causa de abandono do exercício neste estudo, houve outras causas, como as complicações 
cardiovasculares, que representam a principal causa de morbimortalidade nesta população, e a presença de limitações osteomioarticulares (24). É importante destacar que nenhuma das causas de abandono do programa foi relacionada diretamente com a prática do exercício durante as sessões de HD. Embora estes pacientes tenham abandonado o programa, eles o fizeram após um período médio de treinamento de 15 meses, sendo este tempo superior à maioria dos estudos que aplicaram um protocolo de exercício durante a HD (25).

Uma preocupação frequente com a realização de exercício durante as sessões de HD refere-se às possíveis complicações. É sabido que o processo de HD carreia algumas complicações agudas como hipotensão, cãibras musculares, arritmias, náuseas, vômitos, cefaleia, entre outras (26). Estas complicações poderiam ser intensificadas pela prática de exercício, no entanto, em nossa população apenas um paciente apresentou dois episódios de hipotensão severa após o exercício. Como este paciente era diabético, a hipotensão pode ter ocorrido em virtude da disfunção autonômica característica desta doença (26).

No período de cinco anos, com um total de 3.077 sessões individuais de exercício, nosso índice de complicações foi considerado muito baixo. A Alemanha é um país com grande experiência na realização de exercício durante as sessões de HD. 0 grupo responsável por estes programas já totalizou mais de 50 mil sessões individuais de exercício e observou que a prática de exercício durante HD é segura e que as complicações mais comuns foram episódios de cãibras em alguns pacientes (23).

A baixa ocorrência de complicações observada em nosso estudo pode ser atribuída à seleção inicial da amostra, que incluiu pacientes em boas condições clínicas, ao acompanhamento multiprofissional oferecido em nosso centro e à supervisão cuidadosa durante a realização dos exercícios. A supervisão foi realizada por fisioterapeutas, individualmente ou no máximo na proporção de dois pacientes por profissional. Esta estratégia tem sido utilizada por Daul et al. (23), que ressaltam que a supervisão adequada é de grande importância para a baixa ocorrência de complicações durante o exercício realizado nas sessões de HD.

Portanto, conclui-se que a realização de exercício aeróbico durante a sessão de HD é uma prática bem aceita pelos pacientes, segura e não acompanhada de complicações.

\section{Considerações finais}

Uma implicação da prática de exercícios na população geral e em populações de pacientes portadores de doenças crônicas relaciona-se à melhora de parâmetros cardiovasculares, ganho na qualidade de vida e aumento da sobrevida. Considerando-se que a população de renais crônicos apresenta elevados índices de óbitos por doenças cardiovasculares, o estímulo à prática de exercícios se justificaria com base na redução desses eventos e na melhora da qualidade de vida. Neste sentido, em estudo realizado por nosso grupo, a prática de treinamento aeróbico durante as sessões de HD, por um período de três meses, associou-se ao melhor controle da hipertensão arterial e ao aumento na capacidade funcional (13).

Portanto, a adoção desta prática por centros de nefrologia pode constituir uma alternativa terapêutica para a população de pacientes renais crônicos em regime de terapia renal substitutiva e, possivelmente, para pacientes em tratamento conservador.

\section{Agradecimentos}

Aos médicos, à equipe de enfermagem e aos funcionários do Serviço de Hemodiálise do HU - UFJF e da fundação IMEPEN, pela importante assistência durante este trabalho.

Apoio financeiro: Fundação de Amparo à Pesquisa do Estado de Minas Gerais - FAPEMIG (APQ-0245209), Coordenação de Aperfeiçoamento de Pessoal de Nível Superior (CAPES) e Fundação IMEPEN (Instituto Mineiro de Estudos e Pesquisas em Nefrologia).

\section{Referências}

1. Painter PL, Messer-Rehak D, Hanson P, Zimmermann SW, Glass NR. Exercise capacity in hemodialysis, CAPD and renal transplant patients. Nephron. 1986;42(1):47-51.

2. Kouidi E, Albani M, Natsis K, Megalopoulos A, Gigis P, Guiba-Tziampiri 0, et al. The effects of exercise training on muscle atrophy in haemodialysis patients. Nephrol Dial Transplant. 1998;13(3):685-99.

3. Sietsema KE, Hiatt WR, Esler A, Adler S, Amato A, Brass EP. Clinical and demographic predictors of exercise capacity in end-stage renal disease. Am J Kidney Dis. 2002;39(1):76-85. 
4. Painter P, Moore G, Carlson L, Paul S, Myll J, Phillips $\mathrm{W}$, et al. Effects of exercise training plus normalization of hematocrit on exercise capacity and health-related quality of life. Am J Kidney Dis. 2002;39(2):257-65.

5. Sangkabutra T, Crankshaw DP, Schneider C, Fraser $\mathrm{SF}$, Sostaric S, Mason K, et al. Impaired $\mathrm{K}^{+}$regulation contributes to exercise limitation in end-stage renal failure. Kidney Int. 2003;63(1):283-90.

6. Sezer S, Elsurer R, Ulubay G, Ozdemir F, Haberal M. Factors associated with peak oxygen uptake in hemodialysis patients awaiting renal transplantation. Transplant Proc. 2007;39(4):879-82.

7. Painter P. Physical functioning in end-stage renal disease patients: Update 2005. Hemodial Int. 2005; 9(3):218-35.

8. O'Hare AM, Tawney K, Bacchetti P, Johansen KL. Decreased survival among sedentary patients undergoing dialysis: results from the dialysis morbidity and mortality StudyWave 2. Am J Kidney Dis. 2003; 41(2):447-54.

9. Stack AG, Malony DA, Rives T, Tyson J, Murthy BVR. Association of physical activity with mortality in the US dialysis population. Am J Kidney Dis. 2005;45(4): 690-701.

10. Goldberg AP, Hagberg JM, Delmez JA, Florman RW, Harter HR. Effects of exercise training on coronary risk factors in hemodialysis patients. Proc Clin Dial Transplant Forum. 1979;9:39-43.

11. Painter PL, Nelson-Worel JN, Hill MM, Thornbery DR, Shelp WR, Harrington AR, et al. Effects of exercise training during hemodialysis. Nephron. 1986;43(2):87-92.

12. Kouidi E, Grekas D, Deligiannis A, Tourkantonis A. Outcomes of long-term exercise training in dialysis patients: comparison of two training programs. Clin Nephrol. 2004;61(Suppl 1):S31-8.

13. Reboredo MM, Henrique DMN, Faria RS, Chaoubah A, Bastos MG, Paula RB. Exercise training during hemodialysis reduces blood pressure and increases physical functioning and quality of life. Artif Organs. 2010; 34(7):586-93.

14. Miller BW, Cress CL, Johnson ME, Nichols DH, Schnitzler MA. Exercise during hemodialysis decreases the use of antihypertensive medications. Am J Kidney Dis. 2002;39(4):828-33.
15. Vaithilingam I, Polkinghorne KR, Atkins RC, Kerr PG. Time and exercise improve phosphate removal in hemodialysis patients. Am J Kidney Dis. 2004;43(1):85-9.

16. Depaul V, Moreland J, Eager T, Clase CM. The effectiveness of aerobic and muscle strength training in patients receiving hemodialysis and EPO: a randomized controlled trial. Am J Kidney Dis. 2002;40(6):1219-29.

17. Oh-Park M, Fast A, Gopal S, Lynn R, Frei G, Drenth R, et al. Exercise for the dialyzed: Aerobic and strength training during hemodialysis. Am J Phys Med Rehabil. 2002;81(11):814-21.

18. Painter P, Carlson L, Carey S, Paul SM, Myll J. Lowfunctioning hemodialysis patients improve with exercise training. Am J Kidney Dis. 2000;36(3):600-8.

19. Ridley J, Hoey K, Ballagh-Howes N. The exercise-during-hemodialysis program: report on a pilot study. CANNT J. 1999;9(3):20-6.

20. Moug S, Grant S, Creed G, Boulton-Jones M. Exercise during haemodialysis: west of Scotland pilot study. Scott Med J. 2004;49(1):14-7.

21. Borg G. Psychophysical bases of perceived exertion. Med Sci Sports Exerc. 1982;14(5):377-81.

22. Sesso R, Lopes AA, Thomé FS, Bevilacqua JL, Romão Jr. JE, Lugon J. Relatório do censo brasileiro de diálise 2008. J Bras Nefrol. 2008;30(4):233-8.

23. Daul A, Schafers R, Daul K, Philipp T. Exercise during hemodialysis. Clin Nephrol. 2004;61(Suppl 1):S26-30.

24. Sarnak MJ, Levey AS. Epidemiology of cardiac disease in dialysis patients. Semin Dial. 1999;12:69-76.

25. Cheema BSB, Singh MAF. Exercise training in patients receiving maintenance hemodialysis: a systematic review of clinical trials. Am J Nephrol. 2005;25(4):352-64.

26. Himmelfarb J. Hemodialysis complications. Am J Kidney Dis. 2005;45(6):1122-31.

Recebido: 30/01/2011

Received: 01/30/2011

Aprovado: 18/03/2011

Approved: 03/18/2011 